\title{
¿DEBEMOS, PESE A TODO, CRECER? DESAFÍOS AL DECRECIMIENTO COMO HORIZONTE DE EXPECTATIVAS
}

\author{
SHOULD WE, DESPITE EVERYTHING, GROW? CHALLENGES TO DEGROWTH \\ AS A HORIZON OF EXPECTATIONS
}

\author{
DEVEMOS, APESAR DE TUDO, CRESCER? DESAFIOS AO DECRESCIMENTO \\ COMO HORIZONTE DE EXPECTATIVAS
}

\begin{abstract}
JUAN IOSA
https://orcid.org/0000-0001-5590-6043 / juanfiosa@gmail.com Consejo Nacional de Investigaciones Científicas y Técnicas (CONICET), Centro de Investigaciones Jurídicas y Sociales (CIJS) de la Universidad Nacional de Córdoba (UNC), Argentina - CONICET-CIJS-UNC, US21.

Córdoba, Argentina
\end{abstract}

\begin{abstract}
RESUMO
Apresento aqui, apelando para uma discussão local na Argentina, mas fácil de extrapolar para o contexto global, o que chamarei de dilema trágico do crescimento: Se aumentarmos a produção, as exportações e o consumo, é possível, em princípio, reduzir a pobreza e a desigualdade. Mas ao custo de aumentar a perturbação ambiental. Se tomarmos o caminho oposto e favorecermos o decrescimento, poderemos ajudar a prevenir o aquecimento global e a destruição do ecossistema global (Gaia). Mas isso aumentará a pobreza e a desigualdade. Ou crescemos ou diminuímos. Consequentemente, aumentamos a pobreza e a desigualdade ou o aquecimento global e a perturbação ecológica global. Apesar de tudo, parece-me que existem boas razões para advogar o decrescimento. Em todo caso, no final do texto, procuro mostrar que as razões atualmente utilizadas contra o decrescimento não valem como tais.
\end{abstract}

Palavras-chave: crescimento, decrescimento, pobreza, desigualdade, aquecimento global.

\section{ABSTRACT}

Here I present, appealing to a local discussion in Argentina but easy to extrapolate to the global context, what I will call the tragic dilemma of growth: If we increase production, exports, and consumption, it is possible, in principle, to reduce poverty and inequality. The cost of this path is increasing environmental disruption. If we take the opposite path and favor degrowth, we may help prevent global warming and the destruction of the global ecosystem (Gaia). However, this will increase poverty and inequality. We either grow or degrow. Consequently, we either increase poverty and inequality or global warming and global ecological disruption. In spite of everything, it seems to me that there are good reasons to advocate for degrowth. In any case, at the end of the text, I try to show that the reasons currently used against degrowth are not such.

Keywords: growth, degrowth, poverty, inequality, global warming.

\section{RESUMEN}

Aquí presento, apelando a una discusión local Argentina pero inmediatamente extrapolable al contexto global, lo que denominaré el dilema trágico del crecimiento: Si aumentamos la producción, la exportación y el consumo es posible, en principio, disminuir la pobreza y la desigualdad. Pero a costa de incrementar la disrupción ambiental. Si tomamos el camino opuesto y favorecemos el decrecimiento tal vez ayudemos a evitar el calentamiento global y la destrucción del ecosistema global (Gaia). Pero con ello aumentarán la pobreza y la desigualdad. O crecemos o decrecemos. En consecuencia o bien aumentamos la pobreza y la desigualdad o bien el calentamiento global y la disrupción ecológica global. Pese a todo me parece que hay buenas razones para abogar por el decrecimiento. En todo caso al final del texto intento mostrar que las razones corrientemente esgrimidas en contra del decrecimiento no son tales.

Palabras clave: crecimiento, decrecimiento, pobreza, desigualdad, calentamiento global. 


\section{SUMARIO}

INTRODUCCIÓN; 1.EL DILEMA DEL CRECIMIENTO; 2. CRÍTICA A LAS CRITICAS AL DECRECIONISMO; BIBLIOGRAFÍA

\section{INTRODUCCIÓN}

Recientemente Pablo Gerchunoff ha publicado un texto titulado "El Nudo Argentino" en donde plantea una pregunta inquietante: ¿Es posible encontrar una fórmula que reconcilie justicia social y movilidad social? Gerchunoff dedica su artículo a defender la tesis de que para solucionar estos dos grandes problemas Argentina debe crecer y para crecer "es necesario construir una coalición política y social pro exportadora." La pregunta es tan relevante para Argentina como para cualquier otro país. Aquí me centraré en el caso argentino pero creo que las conclusiones (en la medida en que pueda hablarse de tales, aunque tal vez de lo que se trate es de preguntas mejor formuladas) pueden extrapolarse sin mayor problema a otros contextos.

Me interesa distinguir con claridad las ideas de movilidad social ascendente y de justicia social pues quisiera, a modo de introducción a la discusión aquí abordada, reconstruir la idea de Gerchunoff en el sentido de que pese a que hasta el momento "Argentina no ha sabido encontrar la fórmula para firmar un tratado de paz entre $a m b(a) s "$, las dos se verían impulsadas por el crecimiento económico. Gerchunoff, como buen historiador ubica la edad de oro de la movilidad social ascendente en la época de la inmigración del centenario. Al respecto dice que "permea hacia abajo más de lo que se supone pero es, predominantemente, una utopía de clase media, relativamente autónoma del Estado, vinculada al progreso individual y a la modernización colectiva", y que "movilidad social significa crecimiento, competencia, innovación, flexibilidad de la economía y sus instituciones, trabajadores cuyos hijos se convierten en profesionales o empresarios, empresarios que se expanden." Con respecto la justicia social sostiene que es "es una utopía de las clases trabajadoras y, últimamente, de los sectores informales, más dependiente de la intervención pública”, que su sujeto clásico "son los obreros industriales de los años 40” y que "significa salarios altos, protección económica, protección social, dignificación de los desposeídos, empresarios que progresan en sociedad implícita o explícita con el Estado en una comunidad más orgánica."

Veamos ahora cómo Gerchunoff vincula cada una de estas ideas al crecimiento. A su entender para lograr progreso social (o movilidad social ascendente) la Argentina tiene que crecer y para crecer tiene que exportar. De otro modo no entrarían divisas. Sólo si hay más riqueza y más

${ }^{1}$ GERCHUNOFF, P. “El Nudo Argentino”, https://www.eldiplo.org/notas-web/el-nudo-argentino/ 
capital es posible el progreso, la movilidad ascendente de todos los actores. La movilidad a la que refiere Gerchunoff es un subproducto del crecimiento económico. Puede resumirse en la famosa expresión anglosajona "Growth is a rising tide that lifts all boats" ("El crecimiento es una marea ascendente que levanta todos los botes").

Respecto de la relación entre justicia social y crecimiento Gerchunoff afirma que

[...] la justicia social, tal como la practicamos los argentinos, es sinónimo de políticas anti-competitivas y por lo tanto de políticas anti-crecimiento...[que] ahora tenemos una justicia social traída del pasado y que se autodestruye porque bloquea el crecimiento. Argentina es una sociedad bloqueada."

A su juicio, para desbloquear el crecimiento deberíamos, de nuevo, lograr un consenso pro exportador. "La economía argentina necesita productores de dólares en sus campos, en sus industrias, en sus yacimientos mineros y petroleros, en las oficinas de quienes abastecen al mundo de servicios modernos." Y para lograr ese consenso necesitamos cambiar nuestra concepción de justicia social heredada del peronismo: los trabajadores deberían aceptar salarios más bajos en dólares junto a la promesa, por parte de los empresarios, de hacerlos partícipes del posible crecimiento futuro.

Aunque creo que el adjetivo no se le aplica, no me interesa aquí evaluar la solidez de las tesis y argumentos de Gerchunoff. ${ }^{2}$ Sí quisiera subrayar que él, como casi todo el mundo, cree que el crecimiento económico y la exportación son condición necesaria tanto de la movilidad social ascendente como de la justicia social.

Me despido ya de Gerchunoff para seguir mi argumento con dos ideas que, aunque cercanas a las suyas, no son sinónimas. Comencé este texto pensando que lo eran pero el análisis me mostró que no. Las de Pablo obtienen sus nutrientes del campo de la historia mientras que las que quisiera pensar provienen más bien de la economía y la filosofía política. Me refiero a la pobreza y la desigualdad. En todo caso el análisis puede correr por vías paralelas a las hasta recién transitadas: no hay ninguna contradicción interna a estas dos ideas: una sociedad puede crecer disminuyendo la pobreza y la desigualdad al mismo tiempo. De hecho esta era la tesis que Kuznets formulara famosamente en $1955 .^{3}$ Otra cosa distinta es si el crecimiento económico es de hecho condición necesaria para la realización de cada una de estas ideas o si, como creo que es el caso, es posible (aunque improbable) una sociedad menos pobre y más igualitaria aún sin crecimiento.

\footnotetext{
2 Para una lectura muy crítica de las tesis de Gerchunoff consultar SCALETTA, C., "La utopía reaccionaria de exportar salarios bajos" de Disponible on line en https://www.eldiplo.org/notas-web/la-utopiareaccionaria-de-exportar-salarios-bajos

${ }^{3}$ KUZNETS, S. "Economic Growth and Income Inequality", The American Economic Review, Vol. 45, № 1, marzo de 1955, pp. 1 - 28.
} 
¿DEBEMOS, PESE A TODO, CRECER?

Tengamos este problema en mente para más adelante. Antes de avanzar corresponde distinguir estos conceptos.

Mi análisis, al menos por el momento, no requiere más sofisticación que la de sentido común: por desigualdad me refiero, nos referimos, a la posición relativa de los actores sociales, de las clases, en la distribución de la riqueza: Una sociedad desigual es una en que hay mucha distancia relativa entre los que más y los que menos tienen. En cuanto a la pobreza necesito, a los fines de este trabajo, hacer una distinción de nuevo de sentido común: una sociedad es pobre en términos absolutos cuando la riqueza total que acumula es baja en relación al conjunto de su población (y tal vez en relación a la riqueza total de otras naciones comparables). Una sociedad es pobre en términos relativos cuando aquellos que menos tienen son una parte importante de la población. Es así que una sociedad con el $40 \%$ de pobres es pobre en términos relativos pero puede ser rica en términos absolutos si en conjunto acumula mucha riqueza.

Ahora quisiera poner el acento en el hecho, que a pocos pasa desapercibido, que a esta altura del siglo XXI no es posible hablar tan ligeramente de producción, crecimiento y exportación (las condiciones que Gerchunoff ponía como necesarias para lograr la movilidad y la justicia social y que podríamos sin mayor costo trasladar a la solución de la pobreza y la desigualdad) como si fueran ideas de bondad absolutamente incuestionada. Las consecuencias del cambio climático están a la vista y sabemos que la producción de riquezas tanto como su comercialización y consumo depende de la extracción de recursos naturales no renovables y de enormes gastos de energía en sus más variadas formas. La amenaza que nuestro actual modo de producción supone para el planeta en su conjunto es tal que la palabra "antropoceno", de la cual no habíamos oído hablar hasta hace pocos años, está hoy en boca de todos (aunque el corrector automático de Word aún me la marca como error). Estamos, en otras palabras, perfectamente familiarizados con la idea de que la humanidad mediante su actividad se ha convertido en una fuerza geológica capaz de amenazar la vida misma en la tierra y, sin dudas, la supervivencia de la propia especie.

Frente a esta situación se viene proponiendo, al menos desde los años setenta del siglo pasado (estoy pensando en el informe Meadows y en los pioneros textos de Iván Illich), la necesidad del decrecimiento global de la economía: si queremos vivir en un planeta habitable debemos, en conjunto al menos, producir y consumir menos. De ahí que quienes asumen esta idea se opongan sistemáticamente a cualquier proyecto productivo que implique un alto impacto ambiental. Es decir a casi todos. Un botón de muestra para el caso argentino: a comienzos de julio de este año la legislatura fueguina prohibió, sobre la base argumentos pro ambientalistas, la salmonicultura 
en aguas del Canal de Beagle. ${ }^{4}$ Frente a la prohibición las voces críticas de los partidarios del crecimiento no se hicieron esperar. Martín Schapiro, a raíz de la medida, sostuvo que "sin crecimiento es imposible bajar de forma sostenida la pobreza", algo imperioso en un país donde ésta "ha alcanzado hasta el 42 por ciento de la población." En la misma dirección Claudio Scaletta, agregaba: “Para la economía no importa de quién sea el producto ni la naturaleza del bien que se exporte, lo que importa es que generar divisas permite aumentar salarios. Así de simple. Y no generarlas nos conduce al estancamiento y a la pobreza."6 Para Scaletta, tanto como para Schapiro y Gerchunoff, mientras permitan atraer dólares y, consecuentemente, disminuir la pobreza y la desigualdad no parece haber ninguna distinción que hacer entre el recurso objeto de explotación: agronomía, minería e hidrocarburos, pesca, industria, servicios, etc., toda riqueza debe ser extraída.

\section{EL DILEMA DEL CRECIMIENTO}

Estamos entonces frente a lo que cabría denominar el dilema trágico del crecimiento ${ }^{7}$ :

a) Si aumentamos la producción, la exportación y el consumo es posible, en principio, disminuir la pobreza y la desigualdad. Pero a costa de incrementar la disrupción ambiental.

b) Si tomamos el camino opuesto y favorecemos el decrecimiento, tal vez ayudemos a evitar el calentamiento global y la destrucción del ecosistema global (Gaia). Pero con ello aumentarán la pobreza y la desigualdad.

c) 0 crecemos o decrecemos.

d) En consecuencia o bien aumentamos el calentamiento global y la disrupción ecológica global o bien la pobreza y la desigualdad.

${ }^{4}$ https: / / elauditor.info/actualidad/tierra-del-fuego-y-la-ley-que-prohibe-los-criaderos-desalmon_a60edee19be33d30b7799a6dd

${ }_{5}^{5}$ SCHAPIRO, M., "Hay que regular, no prohibir", https://www.eldiplo.org/notas-web/hay-que-regular-noprohibir/

6 SCALETTA, C. "Político y polémico", entrevista disponible en https://almargen.org.ar/2021/07/10/politico-y-polemico/

Para un detallado análisis en defensa de la prohibición de la salmonicultura ver SEMÁN, E., "Matar al Salmón" disponible en https: / / panamarevista.com/matar-al-salmon/?s=08

${ }^{7}$ La calificación puede ser un tanto pomposa pero no creo que sea desatinada. Estamos, efectivamente, ante una tragedia. 
En contra de esta conclusión creo que es posible (aunque improbable) decrecer globalmente disminuyendo (o al menos no aumentando) a la vez la pobreza relativa y la desigualdad. Es cierto que si decrecer es un imperativo debemos aceptar que seremos global e incluso nacionalmente más pobres en términos absolutos (el PBI mundial e incluso el nacional serán más pequeños -aunque no necesariamente todas las naciones deberán decrecer: las más pobres tendrían crédito). Pero esto no significa que aumente la pobreza relativa: la pobreza de los más pobres, aquellos de quienes hablamos cuando decimos que hay hoy un $42 \%$ de pobres en Argentina. No llegaré aquí a sostener esta tesis con todos los argumentos de rigor. Sí intentaré al menos balbucear algunas razones que apuntan en esa dirección. Para ello haré foco en las tesis decrecionistas.

\section{CRÍTICA A LAS CRITICAS AL DECRECIONISMO}

En su reciente libro, luego de una descripción de las transformaciones sociales y económicas de los últimos años, Alejandro Galliano ${ }^{8}$ analiza diversos modelos de futuro posible y deseable (diversas futurabilidades, en los términos de Ezequiel Gatto ${ }^{9}$ ). Me detendré en la reconstrucción de su análisis de la futurabilidad que nos interesa: el decrecionismo.

Galliano comienza su análisis, en los términos que aquí interesan, con la pregunta de André Gorz: ¿Es compatible el equilibrio del planeta con la supervivencia del sistema capitalista?

Para lo que llamaré decrecionismo anticapitalista (o al menos para un decrecionismo; luego podemos pensar la posibilidad de un decrecionismo capitalista, ie., compatible con el capitalismo) la respuesta es no. Por lo tanto debemos decrecer y abandonar el capitalismo. Podemos poner el argumento decrecionista anticapitalista de nuevo en forma silogística:

a) Para el equilibrio del planeta es condición necesaria el decrecimiento de la producción material.

b) Para el funcionamiento del capitalismo es necesario el crecimiento económico continuo.

c) $\mathrm{O}$ bien hay o decrecimiento o bien hay crecimiento.

d) Por lo tanto o bien hay equilibrio del planeta o bien hay capitalismo. 0 lo que es lo mismo, el capitalismo es incompatible con el equilibrio planetario.

\footnotetext{
${ }^{8}$ GALLIANO, A., 2020: ¿Por qué el Capitalismo puede soñar y nosotros no? Buenos Aires, Siglo XXI Editores.

${ }^{9}$ GATTO, E., 2018: Futuridades, Rosario, Casagrande.
} 
e) El equilibrio planetario es condición de la vida humana en la tierra, por lo menos a largo plazo.

f) Valoramos o tenemos un interés inderrotable en el sostenimiento de las condiciones de posibilidad de la vida humana (y de la vida en su conjunto) a largo plazo.

g) Por lo tanto el curso racional es decrecer abandonando el capitalismo.

Si no nos gusta la conclusión debemos atacar alguna de sus premisas. Las más directamente expuestas a la crítica son las premisas a) y b) del último argumento. Se podría negar que el decrecimiento sea condición necesaria del equilibrio o que el crecimiento sea condición necesaria del capitalismo. Pero las razones a favor de a) son bastante sólidas: a tasas medias o altas de crecimiento a largo plazo el capitalismo es insostenible en virtud del seguro colapso de su base material: a esas tasas, por ejemplo, no se lograría la decarbonización de la atmósfera y cabría esperar las peores consecuencias del calentamiento global y del agotamiento de los recursos naturales.

Por su parte, la verdad de la tesis b); ie., la cuestión de si es o no posible el capitalismo sin crecimiento, depende tanto de cuestiones conceptuales como empíricas, es decir, depende tanto de qué significa capitalismo como de cuáles sean las condiciones materiales de reproducción de este sistema. La posibilidad empírica del sostenimiento del capitalismo a tasas de crecimiento muy bajas, nulas o negativas me parece más interesante que la cuestión conceptual. De hecho Galliano afirma que “...el sistema capitalista... en teoría podría sobrevivir sin crecimiento” 10 , lo que equivale a afirmar su posibilidad junto a su improbabilidad empírica (a esto me refería cuando hablaba de decrecionismo capitalista). En todo caso un trabajo que pretendiera dar acabada respuesta a los interrogantes aquí planteados debería listar y evaluar una por una las referidas condiciones conceptuales y empíricas. Recién entonces estaría en condiciones de afirmar o negar la sostenibilidad a largo plazo del capitalismo sin crecimiento. No cuento hoy con las herramientas analíticas para emprender ese camino. Me centraré en cambio en las objeciones de Galliano al decrecimiento y trataré de evaluarlas. Pero antes quisiera introducir dos distinciones.

Primero, el decrecionismo puede concebirse como una doctrina acerca de cierto estado de cosas deseable, ie, una doctrina sobre los fines. Podríamos calificar de decrecionista a una teoría que resalte el valor último de la preservación y el florecimiento de la vida humana y, en última instancia, de toda vida sobre la tierra, aun a costa de menos riqueza material. El decrecionismo también puede pensarse como una doctrina sobre los medios necesarios para

${ }^{10}$ GALLIANO, A., cit., 86. 
alcanzar ese estado de cosas deseable. 0 , por último, como ambas cosas. En cualquier caso hay que destacar que las críticas dirigidas a los medios no cancelan los fines. Además los medios son contingentes: demostrado que cierto medio no es apto para lograr el fin buscado, bien cabe buscar medios alternativos. Salvo, claro está, que se demuestre que no hay ningún medio apropiado para obtener el fin en cuestión.

Segundo, el decrecionismo se propone por un lado como una doctrina política que señala un estado de cosas que debe ser deliberadamente buscado, como el fruto de una búsqueda colectiva consciente. Pero también se presenta como la consecuencia necesaria de la dinámica económica misma: para muchos economistas mainstream el mundo entró en un período de estancamiento secular. El mundo va a decrecer sí o sí: basta esperar, por ejemplo, el agotamiento del petróleo o del resto de los recursos naturales. Bajo esta perspectiva el decrecionismo es más bien una doctrina sobre cómo posicionarse colectivamente frente a este hecho.

Con estas distinciones en mano podemos encarar mejor la cuestión de cuáles son las objeciones de Galliano al decrecionismo.

1) El decrecionismo es inviable: "La indefinición del decrecionismo sobre el capitalismo no lo inmuniza contra el gran problema de todos los movimientos anticapitalistas ¿Cómo llevar a la práctica estas ideas?"11 Alejandro ve inviables las propuestas políticas decrecionistas pues su adopción es muy improbable en el marco del tipo de preferencias comunes en la sociedad capitalista.

Pero esta es una objeción a los medios propuestos. Y sobre los medios debemos ser imaginativos y en todo caso dar la batalla política si es que los fines son efectivamente tales. Además esta objeción se presenta ante el decrecionismo como programa político ex ante el estancamiento económico, no al decrecionismo como "tutorial postapocalíptico", ${ }^{12}$ como programa político que asume el estancamiento e incluso el colapso sistémico como un dato, como un hecho. ¿Qué políticas sería razonable adoptar si la economía mundial no fuera a crecer más?

Ya sea ex ante o ex post el decrecionismo apunta a la construcción de una sociedad con “un menor metabolismo, pero más importante aún, un metabolismo con una estructura diferente."13 Básicamente lo que necesitamos es una sociedad menos productiva, más pobre (en sentido absoluto), y donde lo producido esté mejor distribuido. Este fin se puede alcanzar, o esta situación de menor productividad se puede enfrentar, con diferentes herramientas. Las propuestas son contradictorias: algunas apuntan a la planificación central, ie., estatal, otras son más bien

\footnotetext{
11 Ídem.

12 GALLIANO, A., cit., 89.

13 Idem.
} 
anarquistas. Ambas propuestas chocan con el ethos de la sociedad capitalista: "los estímulos no monetarios pesan cada vez menos en la conducta de las personas. Proponerle decrecer a una sociedad así es suicidarse políticamente." 14 Pero estos son problemas de medios, y sólo válidos como tales para programas ex ante. ¿Qué otros problemas enfrenta el decrecionismo?

2) El decrecionismo es injusto:

La tragedia decrecionista es que su propuesta choca contra sus propios valores: en los países ricos es incompatible con la democracia, en los países pobres es directamente injusta. Sin distribución del ingreso, detener el crecimiento en este momento implicaría dejar bajo el nivel de la pobreza al $25 \%$ de la población mundial. ${ }^{15}$

No resulta claro por qué Alejandro piensa que la distribución del ingreso no es parte del programa decrecionista. Me parece que cualquier decrecionismo sensato la incluiría. Justamente, una distribución masiva del ingreso es lo que permitiría, en un marco de mayor pobreza absoluta, sostener índices sensatos de pobreza relativa. Sin duda estas ideas pueden tacharse de naif o utópicas. Pero de nuevo: ese calificativo cabe solo ex ante.

También está la cuestión de la brecha de desarrollo entre países ricos y pobres. Pedirles a todos por igual que se abstengan de crecer sería condenar a los países subdesarrollados a una situación de perpetua pobreza: "una auténtica patada a la escalera para que los de abajo no suban."16 Pero los teóricos del decrecimiento están pensando estrategias de compensación. Esto sucede por ejemplo con el tema de la huella de carbono. Los modelos que se manejan dan más libertad de producción de carbono a los países pobres con el objeto de cerrar la brecha.

3) El decrecionismo es reaccionario: "gran parte de la imaginación política decrecionista parece cifrarse en [una] concepción primitivista del futuro."17 Por atrás habría una secreta esperanza en que todos podamos ser buenos salvajes roussonianos, "una confianza en la sabiduría ancestral de cosmovisiones (primitivas). ${ }^{18}$ " Este primitivismo implicaría un rechazo de cualquier sistema energético y, en última instancia una tecnofobia: Ivan Illich. Pero no está claro que todo decrecionismo sea tecnófobo ni que toda crítica de la técnica por alienante sea equivalente a tecnófoba. Tampoco está claro que "vivir con menos" equivalga a un regreso a las cavernas o, más acá, que todos nos pongamos a cultivar nuestro propio jardín. El modo adecuado de producción

\footnotetext{
14 GALLIANO, A., cit., 90 - 91.

15 GALLIANO, A., cit., 91.

16 GALLIANO, A., cit., 92.

17 GALLIANO, A., cit., 93.

18 Idem.
} 
¿DEBEMOS, PESE A TODO, CRECER?

de lo realmente necesario es algo que sólo se podrá determinar a posteriori, luego de los estudios empíricos del caso que determinen el umbral de producción sustentable.

4) El decrecionismo pide demasiado de las personas y las sociedades:

El decrecionismo es políticamente prometeico y materialmente conservador: concibe al planeta como un recurso limitado y fragilísimo pero espera de la sociedad una plasticidad infinita para modificar sus hábitos. Es una contradicción que los conservadores han detectado con agudeza. ${ }^{19}$

No se ve cuál sea la contradicción referida. La sociedad y la naturaleza no operan bajo las mismas leyes.

En todo caso, ¿qué tan antidecrecionista es Galliano? En principio parece que mucho. Al final del capítulo en cuestión afirma:

Quizás el elemento más trágico del primitivismo decrecionista sea la parálisis política a la que se condena. El decrecionismo aporta un diagnóstico impecable sobre el agotamiento de los recursos impulsado por un capitalismo que necesita quemar todo para producir algo. No hay manera de relativizar o deconstruir el dato material, objetivo, de la crisis climática. El llamado a la acción es impostergable. Pero la reivindicación de modelos sociales primitivos, la mistificación de la pobreza y la confianza, casi totalitaria, en que la sociedad se adaptará a ellos es socialmente injusta, ideológicamente peligrosa y políticamente inútil: no va a ocurrir, no importa cuánto daño hagamos en el camino. ${ }^{20}$

El pasaje es contundente. Pareciera seguirse que debemos tirar el decrecionismo al basurero de las futurizaciones impracticables y solo atractivas en apariencia.

Pero Alejandro a renglón seguido sostiene:

[...] ...es precisamente la planificación racional de la economía lo que puede modificar el metabolismo social sin acabar en una celebración de la miseria y el atraso. El mayor consumo de recursos se concentra en los países más ricos del mundo y en los ciudadanos más ricos de cada país. La solución pasa por gobernar a esa clase y a esa desigualdad usando las herramientas consagradas por la modernidad: leyes y tecnología. ${ }^{21}$

En la misma dirección, al cerrar su libro con lo que podría contar como su propia futurabilidad, Alejandro afirma:

El capitalismo 4.0 es un sistema sumamente inestable que depende de diferentes formas extractivas para funcionar, que amplía la masa marginal y requiere de condiciones cada vez más excepcionales y violentas para funcionar. En algún momento colapsará o se reordenará en un capitalismo 5.0. 0 ambas cosas. No tenemos la fuerza para vencerlo y salir de él es imposible. Debemos gobernarlo: aprovecharlo donde sea necesario, combatirlo donde sea nocivo y regularlo donde

\footnotetext{
${ }^{19}$ GALLIANO, A., cit. 95.

20 GALLIANO, A., cit. 96.

21 Ídem.
} 
sea insuficiente. Y, sobre todo, parasitarlo allí donde podamos: luchar por el ocio civilizatorio y por el control social de las rentas naturales, digitales y financieras, tanto para captarlas y redistribuirlas como para limitarlas. En fin, contribuir a la muerte lenta del capitalismo 4.0. ${ }^{22}$

Galliano no está en contra del decrecionismo. Por el contrario, asume una forma particular de futurabilidad decrecionista. Quizás asumir con él ese horizonte de expectativas sea un buen lugar para seguir pensando juntos. Quizás cuidar el planeta sea lo mismo que dejar de explotarnos tan salvajemente entre nosotros.

\section{BIBLIOGRAFÍA}

GALLIANO, A., 2020: ¿Por qué el Capitalismo puede soñar y nosotros no? Buenos Aires, Siglo XXI Editores. GATTO, E., 2018: Futuridades, Rosario, Casagrande.

GERCHUNOFF, P. El Nudo Argentino, en https://www.eldiplo.org/notas-web/el-nudo-argentino/

KUZNETS, S. "Economic Growth and Income Inequality", The American Economic Review, Vol. 45, № 1, marzo de 1955, pp. 1 - 28.

SCALETTA, C., La utopía reaccionaria de exportar salarios bajos, en https://www.eldiplo.org/notasweb/la-utopia-reaccionaria-de-exportar-salarios-bajos/

SCALETTA, C. Político y polémico, entrevista disponible en https://almargen.org.ar/2021/07/10/politicoy-polemico/

SCHAPIRO, M., Hay que regular, no prohibir, en https://www.eldiplo.org/notas-web/hay-que-regular-noprohibir/

SEMÁN, E., Matar al Salmón, en https://panamarevista.com/matar-al-salmon/?s=08

Artigo convidado

COMO FAZER REFERÊNCIA AO ARTIGO (ABNT):

IOSA, Juan. ¿Debemos, pese a todo, crecer? desafíos al decrecimiento como horizonte de expectativas.

Revista Eletrônica do Curso de Direito da UFSM, Santa Maria, RS, v. 15, n. 3, e67873, set./dez. 2020.

ISSN 1981-3694. DOI: http://dx.doi.org/10.5902/1981369467873. Disponível em:

https://periodicos.ufsm.br/revistadireito/article/view/67873 Acesso em: dia mês. ano.

Direitos autorais 2020 Revista Eletrônica do Curso de Direito da UFSM

Editores responsáveis: Rafael Santos de Oliveira e Angela Araujo da Silveira Espindola

\section{(i) $(9)$}

Esta obra está licenciada com uma Licença Creative Commons Atribuição-NãoComercial-SemDerivações 4.0 Internacional.

\section{SOBRE O AUTOR}

\section{JUAN IOSA}

Profesor de Filosofia del Derecho de la Universidad Nacional de Córdoba y profesor de Teoría de la

Argumentación

22 GALLIANO, A., Cit., 174. 